\title{
Viability of Compressed Sensing as a Dose Reduction Strategy in STEM
}

\author{
W. Van den Broek, ${ }^{1 *}$ B.W. Reed, ${ }^{2}$ A. Béché, ${ }^{3}$ J. Verbeeck, ${ }^{3}$ and C.T. Koch $^{1}$ \\ 1. Institut für Physik \& IRIS Adlershof, Humboldt-Universität zu Berlin, Berlin, Germany \\ 2. Integrated Dynamic Electron Solutions, Inc., Pleasanton CA, US \\ 3. EMAT, Antwerp University, Antwerp, Belgium \\ * Corresponding author: vandenbroek@physik.hu-berlin.de
}

The goal of dose reduction in scanning transmission electron microscopy (STEM) is to reduce electron beam damage to the specimen as the latter is often related to the total dose [1,2]. Other damage mechanisms such a dose rate effects or the spatial distribution of the dose are not considered here. Compressed sensing (CS) [3] is a recording procedure that, through application of an accompanying reconstruction technique, is capable of retrieving a signal from a surprisingly low amount of measurements. It has thus been considered as a candidate for dose reduction in STEM. However, since the mere number of measurements does not necessarily guarantee a lower dose, it is examined in this work if a CS reconstruction can outperform a conventional Shannon scan that is recorded with the same electron budget and denoised based on the same prior knowledge applied in the CS reconstruction. An important difference with much of CS literature is the use of positive, flux-preserving sensing matrices and the presence of Poisson noise.

In CS, the $M$ measurements $y$ are linear combinations of the unknown signal $x: y=I A x$. Here, $I$ is the beam intensity, the $N$ by 1 vector $x$ is the image written in long vector format, $A$ is the $M$ by $N$ sensing matrix, and $M<N$. By setting a randomly chosen fraction $p$ of the elements of each row of $A$ to $1 /(p N)$ and the other elements to zero, recordings with constant dose are ensured. Setting $p=1 / N$ yields the socalled inpainting set-up [4]. A Shannon scan with equal dose is implemented as a diagonal $N$ by $N$ matrix $A$ with non-zero elements equal to $M / N$. The signal $x$ is then retrieved by minimizing its total generalized variation, subject to the mean square residuals equaling the noise variance [5]. By swapping out the CS sensing matrix for that of the Shannon scan a denoising with the same prior knowledge is realized.

In order to compare different settings, we define the detective quantum efficiency: $\mathrm{DQE}=a \mathrm{SNR}^{2} \mathrm{y} /$ $\mathrm{SNR}^{2}{ }_{\text {in, }}$, where $\mathrm{SNR}_{\mathrm{y}}$ and $\mathrm{SNR}_{\text {in }}$ are the signal-to-noise ratios of the signal $y$ and the incoming signal, respectively, while $a$ reflects the different number of measurements in both cases, hence $a=M / N$ for CS and $a=1$ for the Shannon scan. Following [5] these DQEs can be calculated as a function of $p$ explicitly, yielding $(1-p) /(p N)$ and 1 for CS and Shannon scans respectively, in the presence of Poisson noise and the absence of read-out noise. It is shown [5] that the DQE tracks the amount of Fisher information in the measurements excellently. Next, it is illustrated that the DQE predicts the minimum of the mean squared error (MSE) of the reconstructions well, and that it can hence be used to predict optimal values for $p$ and compare different set-ups.

A gold nanorod was recorded on a FEI Titan ${ }^{3}$ microscope operating in STEM mode at $300 \mathrm{kV}$. The experiment exhibits almost pure Poisson noise. First, an image was raster scanned with a high intensity and after application of a 3 by 3 median filter, it served as ground truth to compute the MSE. Then an image was recorded with a 35 times lower dose and from it 9 inpainting recordings were extracted with $M$ ranging from $0.1 N$ to $0.9 N$; from these recordings a reconstruction was computed. For comparison a denoising was calculated as well. In Figure 1 the recordings and selected reconstructions are presented, 
and it is shown that the MSE follows the predicted DQE of $M / N$ well.

Since the DQE for CS is maximized for $p=1 / N$, an inpainting approach is the optimal CS set-up under Poisson noise and in the absence of read-out noise. Furthermore, for this value of $p$ the DQE for CS is virtually equal to the DQE of a Shannon scan, leading to the conclusion that no gain in Fisher information nor an improvement of reconstruction quality (measured through the MSE) is to be expected from CS, as was corroborated by the experiment. [6]

\section{References:}

[1] R. Henderson, Quarterly Reviews of Biophysics 28 (1995), p. 171.

[2] D. Shi, et al., eLife 2 (2013), p. e01345.

[3] J. Romberg, IEEE Signal Processing Magazine 25 (2008), p. 14.

[4] K. Papafitsoros and C.B. Schönlieb, Journal of Mathematical Imaging and Vision 48 (2014), p. 308.

[5] W. Van den Broek et al., IEEE Transactions on Computational Imaging, DOI:

10.1109/TCI.2019.2894950

[6] Acknowledgements. WVdB: DFG project BR 5095/2-1. Hercules fund from the Flemish Government for the Qu-Ant-EM microscope. AB, JV: FWO project G093417N. CTK: DFG project CRC 951. Prof. L. Liz-Marzan for the TEM sample.
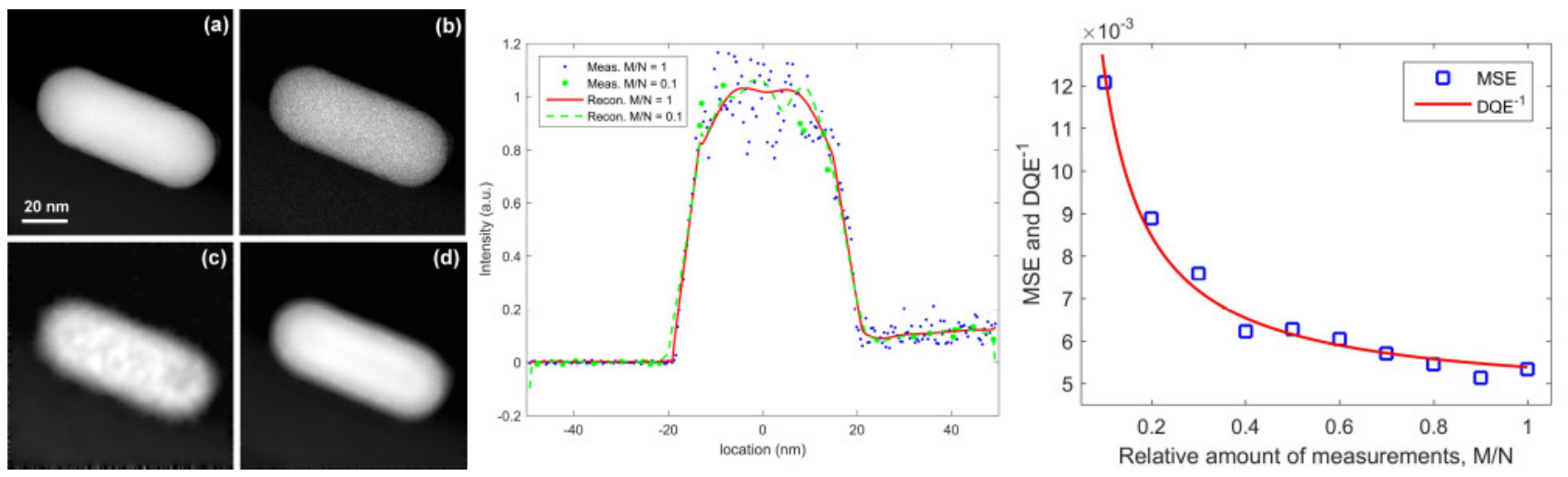

Figure 1. Left: Experimental ADF-STEM data. (a) Ground truth image. (b) Image at 35 times lower dose. (c) Reconstruction from $M=0.1 N$ measurements. (d) Reconstruction from $M=N$ measurements, i.e. a denoising. Middle: Profiles and measurements for the central vertical line of the reconstructions, for $M=0.1 N$ and $M=N$. Right: After a linear transformation, the $\mathrm{DQE}^{-1} \mathrm{~s}$ match well to the MSEs of the experimental reconstructions. 